\title{
Stress increases intracardiac 4D flow cardiovascular magnetic resonance -derived energetics and vorticity and relates to $\mathrm{VO}_{2} \max$ in Fontan patients
}

Vivian P. Kamphuis ${ }^{1,2}$, Mohammed S. M. Elbaz ${ }^{3}$, Pieter J. van den Boogaard ${ }^{4}$, Lucia J. M. Kroft ${ }^{4}$, Hildo J. Lamb ${ }^{4}$, Mark G. Hazekamp ${ }^{5}$, Monique R. M. Jongbloed ${ }^{6}$, Nico A. Blom ${ }^{1,7}$, Willem A. Helbing ${ }^{8,9}$, Arno A. W. Roest ${ }^{*+}$ (D) and Jos J. M. Westenberg ${ }^{3+}$

\begin{abstract}
Background: We hypothesize that dobutamine-induced stress impacts intracardiac hemodynamic parameters and that this may be linked to decreased exercise capacity in Fontan patients. Therefore, the purpose of this study was to assess the effect of pharmacologic stress on intraventricular kinetic energy (KE), viscous energy loss (EL) and vorticity from four-dimensional (4D) Flow cardiovascular magnetic resonance (CMR) imaging in Fontan patients and to study the association between stress response and exercise capacity.

Methods: Ten Fontan patients underwent whole-heart 4D flow CMR before and during $7.5 \mu \mathrm{g} / \mathrm{kg} / \mathrm{min}$ dobutamine infusion and cardiopulmonary exercise testing (CPET) on the same day. Average ventricular KE, EL and vorticity were

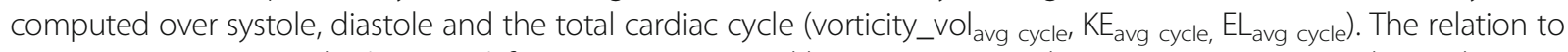
maximum oxygen uptake $\left(\mathrm{VO}_{2}\right.$ max) from CPET was tested by Pearson's correlation or Spearman's rank correlation in case of non-normality of the data.
\end{abstract}

Results: Dobutamine stress caused a significant $88 \pm 52 \%$ increase in KE (KE avg cycle: $1.8 \pm 0.5$ vs $3.3 \pm 0.9 \mathrm{~mJ}, P<0.001$ ), a significant $108 \pm 49 \%$ increase in EL ( $E L_{\text {avg cycle: }} 0.9 \pm 0.4 \mathrm{vs} 1.9 \pm 0.9 \mathrm{~mW}, P<0.001$ ) and a significant $27 \pm 19 \%$ increase in vorticity (vorticity_volavg cycle: $3441 \pm 899 \mathrm{vs} 4394 \pm 1322 \mathrm{~mL} / \mathrm{s}, P=0.002$ ). All rest-stress differences (\%) were negatively correlated to $\mathrm{VO}_{2} \max \left(\mathrm{KE}_{\text {avg cycle: }} r=-0.83, P=0.003\right.$; ELavg cycle: $r=-0.80, P=0.006$; vorticity_volavg cycle: $r=-0.64, P=$ 0.047).

Conclusions: 4D flow CMR-derived intraventricular kinetic energy, viscous energy loss and vorticity in Fontan patients increase during pharmacologic stress and show a negative correlation with exercise capacity measured by $\mathrm{VO}_{2} \mathrm{max}$.

Keywords: 4D flow CMR, Flow, Kinetic energy: energy loss, Vorticity, Fontan

\section{Background}

The Fontan procedure is a palliative surgical procedure for patients with complex congenital intracardiac deformations in whom a biventricular circulation cannot be created [1]. Survival of patients after the procedure has increased drastically over the past decades [2,3], but still,

\footnotetext{
* Correspondence: a.roest@lumc.nl

${ }^{+}$Arno AW Roest and Jos JM Westenberg are shared last authorship

'Department of Pediatrics division of Pediatric Cardiology, Leiden University Medical Center, Leiden, the Netherlands

Full list of author information is available at the end of the article
}

these patients exhibit diminished exercise capacity, which is related to a worse functional health status [4-6]. Several factors have been identified that play a role in the reduced exercise capacity in these patients, such as an inadequate ventricular response to exercise, reduced pulmonary and pulmonary vascular functions, muscle weakness and energy loss in the Fontan tunnel [7-12]. Four-dimensional (4D) flow cardiovascular magnetic resonance (CMR) imaging enables comprehensive assessment of in vivo blood flow patterns and quantification of hemodynamic parameters from blood flow velocity such as kinetic energy (KE),

(c) The Author(s). 2019 Open Access This article is distributed under the terms of the Creative Commons Attribution 4.0 International License (http://creativecommons.org/licenses/by/4.0/), which permits unrestricted use, distribution, and 
viscous energy loss (EL, the KE that is lost due to viscosity-induced frictional forces) and vorticity (a measure of the local spinning of blood particles) [13]. Previous studies have suggested that such vortical spinning patterns of blood flow (vortex formation) may help preserve kinetic energy by minimizing energy losses, hence enabling energy-efficient blood transportation [14, 15].

Recently, altered intracardiac flow patterns, levels of kinetic energy and vortex formation in the intraventricular blood flow pattern were observed in Fontan patients [16-18]. Furthermore, reduced exercise capacity was linked to EL in the total cavopulmonary connection (TCPC) of Fontan patients [10, 12]. However, currently the link between altered intracardiac hemodynamics during stress and exercise capacity remains unknown. We hypothesize that dobutamine-induced stress would impact these intracardiac hemodynamic parameters and that this may be linked to decreased exercise capacity in Fontan patients.

Therefore, the purpose of this study was 1) to non-invasively assess the influence of pharmacologic stress on $\mathrm{KE}$, EL and vorticity measured by $4 \mathrm{D}$ flow CMR in intraventricular blood flow in Fontan patients and 2) to study the association between the hemodynamic stress response in terms of KE, EL and vorticity and exercise capacity assessed by maximum oxygen uptake $\left(\mathrm{VO}_{2}\right.$ max) from cardiopulmonary exercise tests (CPET).

\section{Methods}

\section{Study population}

Twenty-six Fontan patients were prospectively evaluated in this study at the Leiden University Medical Center (LUMC) as part of a multicenter study that was approved by the Medical Ethical Committee of the Erasmus Medical Center in Rotterdam (MEC-2014326, NL48188.078.14), with local approval of the Medical Ethical Committee of the LUMC, The Netherlands. Informed consent was obtained from all of these participants. All methods were performed in accordance with the relevant guidelines and regulations. All patients underwent CPET and a CMR on the same day. Patients were included in the present study when the dobutamine stress CMR scan with 4D flow CMR at rest and stress was fully completed: which was the case in 10/26 (38\%) patients. Reasons for not finishing the complete scan were: 10 (38\%) patients refused stress testing, in 4 (15\%) patients stress testing could only be completed without 4D flow CMR and in 2 (8\%) patients dobutamine infusion had to be stopped because of ventricular extra systoles.

\section{Cardiopulmonary exercising testing}

Cardiopulmonary exercising testing was performed on an upright bicycle ergometer (General Electric Healthcare,
Waukesha, Wisconsin, USA). Starting wattage and workload increase per minute were based on patient's baseline condition and defined by the attending physician. Patients were encouraged to exercise until exhaustion. $\mathrm{VO}_{2} \max$ was derived from the CPET following previously published methods [19].

\section{Cardiovascular magnetic resonance acquisition and image analysis}

A complete CMR scan including whole-heart 4D flow CMR was obtained on a $3 \mathrm{~T}$ scanner (Ingenia, Philips Healthcare, Best, The Netherlands) during rest and with dobutamine $7.5 \mu \mathrm{g} / \mathrm{kg} / \mathrm{min}$. Details on CMR acquisition are provided in Appendix 1. Retrospective gating was used with 30 phases reconstructed to represent one cardiac cycle. Free breathing was allowed without using motion suppression such as gating by navigator or bellow, but we acquired three signal averages to minimize effects of breathing motion. Dobutamine infusion was reduced to $5 \mu \mathrm{g} / \mathrm{kg} / \mathrm{min}$ or stopped in case of a $>50 \%$ increase in heart rate (HR), systolic, or diastolic blood pressure or when significant rhythm disorders occurred. The test was stopped when the patient experienced discomfort. Contour segmentation was performed using inhouse developed MASS software (Medis Medical Imaging Systems, Leiden, The Netherlands) by one observer (VPK) with over 3 years of experience in CMR and verified by a radiologist (LJMK) with over 20 years of experience in CMR. Ventricular volume was segmented in all slices and phases in the cine transversal images (papillary muscles were included in the ventricular volume; remaining parts of the septum in "biventricular" patients were not included in the ventricular volume). Segmented volumes were then used to compute stroke volume (SV) as: end-diastolic volume - end-systolic volume. Cardiac output (CO) was computed as $S V \times H R$. We used the sphericity index to measure the change in the shape of the ventricle between rest and stress. The sphericity index was calculated as the short to long axis ratio: ventricular width/ventricular height at end-systole and enddiastole in the 2D cine transversal images. Time points of systolic and diastolic phases were derived from the flow-time curves that resulted from retrospective valve tracking assessing the inflow and outflow of the systemic ventricle in Fontan patients, following previously published methods [20]. Segmentation of the ventricular cavity in the 4D Flow CMR acquisition, that is required for the energy and vorticity analysis, was obtained by transforming the available time-varying segmentation of multi-slice cine transversal anatomical acquisition to the 4D Flow CMR data [21]. This procedure was performed using in-house developed MASS software by one observer (VPK) with over 3 years of experience in CMR and verified by researcher (MSME) with 6 years of experience in 
CMR. Aliasing of 4D Flow velocity images was visually checked in the source images (all three velocity components) and unwrapped using a sliding velocity scaling in MASS software. Following previous work [18, 21-23], to account for potential patient-motion related misalignment between the 4D flow CMR and transversal anatomical cine acquisitions, automated image-based 3D rigid registration by mutual information was performed using the phase with the maximal depiction of the ventricular cavity in both scans with the Elastix image registration toolbox [24].

\section{Computation of KE, EL and vorticity in the 4D flow CMR data}

The amount of intraventricular KE was computed as $1 / 2$ $m v^{2}$, with $m$ as the mass representing the voxel volume multiplied by the density of blood $(1.025 \mathrm{~g} / \mathrm{mL})$ and $v$ as the magnitude of the 3-directional velocity vector acquired from 4D flow CMR. For each acquired timephase, volumetric KE was then computed by integrating (by cumulative sum) the computed KE over the segmented 3D ventricular volume. The KE was quantified by the time-average kinetic energy over systole $\left(\mathrm{KE}_{\mathrm{avg}}\right.$ systole $)$, diastole $\left(\mathrm{KE}_{\mathrm{avg} \text { diastole }}\right)$ and the complete cardiac cycle $\left(\mathrm{KE}_{\text {avg cycle }}\right)$, all expressed in millijoule $(\mathrm{mJ})$ [23]. EL was computed from 4D flow CMR using the dissipation terms from the Navier-Stokes energy equations, following recently published method [21, 23], assuming blood as a Newtonian fluid. Average EL in milliWatt $(\mathrm{mW})$ was computed over systole $\left(\mathrm{EL}_{\mathrm{avg} \text { systole }}\right)$, diastole $\left(\mathrm{EL}_{\text {avg diastole }}\right)$ and the complete cardiac cycle $\left(\mathrm{EL}_{\text {avg cycle }}\right)$.

The vorticity metric (mathematically computed as the curl of velocity field - here derived from 4D flow CMR) enables direct quantification of the strength of vortical flow at every voxel (i.e. voxel-wise vorticity) as well as the integrating (total sum) of the total vorticity over the entire chamber volume (i.e. indicated here as vorticity vol). More technical details can be found in: [25]. Voxelwise vorticity magnitude (in $1 / \mathrm{s}$ ) was computed for each acquired time-phase and then integrated over the total ventricular volume [23]. To differentiate from voxel-wise vorticity, we will refer to this integrated volumetric vorticity as vorticity_vol (in $\mathrm{mL} / \mathrm{s}$ ). This vorticity_vol is a scalar quantity and does not take the vorticity direction into account. In order to quantify the integral vorticity vol, the time-averaged vorticity over systole and diastole and the total cardiac cycle (vorticity_vol ${ }_{\text {avg systole, vorti- }}$ city_vol $_{\text {avg diastole, }}$ vorticity_vol ${ }_{\text {avg cycle }}$ respectively) were assessed. All EL, KE and vorticity parameters at rest and stress are also given normalized by SV.

\section{Statistical analysis}

Data analysis was performed using SPSS Statistics (version 23.0 Statistical Package for the Social Sciences
(SSPS), International Business Machines, Inc., Armonk, New York, USA). Variables were tested for normal distribution using the Shapiro-Wilk test. Continuous data is reported as mean \pm standard deviation (SD) or median [interquartile range (IQR)], in case of non-normality of the data. The absolute differences between rest and stress measurements (stress measurement - rest measurement) were assessed and significance was tested with the paired samples t-test or the Wilcoxon signedrank test in case of non-normality of the data. Furthermore, the relative differences (as a percentage) between rest and stress measurements [((stress measurement rest measurement)/rest measurement $) \times 100]$ were calculated. Correlation between measurements was tested with the Pearson correlation coefficient or the Spearman's rank correlation coefficient in case of non-normality of the data. Correlation was classified as follows: > 0.95: excellent; 0.95-0.85: strong; 0.85-0.70: good; 0.700.5 : moderate; $<0.5$ : poor. A $P$-value $<0.05$ was considered statistically significant.

\section{Results}

Characteristics of the Fontan patients are shown in Table 1. HR increased from $83.4 \pm 18.9 \mathrm{bpm}$ to $110.9 \pm$ $20.0 \mathrm{bpm}$ between the rest and dobutamine stress scans $(P<0.001)$. Left ventricular end-diastolic volume and sphericity indices did not change significantly from rest to stress $(P \geq 0.12)$. Stroke volume increased from $80.8 \pm 29.4$ to $92.0 \pm 33.9 \mathrm{~mL}$ between the rest and dobutamine stress scans $(P=0.004)$. In one patient, dobutamine infusion was decreased to $5 \mu \mathrm{g} / \mathrm{kg} / \mathrm{min}$ because of a $>50 \%$ increase in systolic blood pressure from baseline.

\section{Intraventricular KE, EL and vorticity_vol at rest and stress} Table 2 shows the non-normalized results of KE, EL and vorticity_vol at rest and dobutamine stress, for the complete cardiac cycle but also averaged for systole and diastole. Average KE significantly increased by $88 \pm 52 \%$ during the complete cardiac cycle $(1.8 \pm 0.5$ vs $3.3 \pm 0.9$ $\mathrm{mJ}, P<0.001)$. With dobutamine stress, average EL significantly increased by $108 \pm 49 \%$ when calculated over the complete cardiac cycle $(0.9 \pm 0.4$ vs $1.9 \pm 0.9 \mathrm{~mW}$, $P<0.001)$. Average vorticity_vol significantly increased by $27 \pm 19 \%$ when calculated over the complete cardiac cycle $(3441 \pm 899$ vs $4394 \pm 1322 \mathrm{~mL} / \mathrm{s}, P=0.002)$.

Figure 1 shows an example of the intracardiac hemodynamics during stress in one patient. Boxplots of the absolute differences for the total group and the individual changes between rest and stress values of $\mathrm{KE}$, EL and vorticity_vol are shown in Fig. 2 and Table 3 in Appendix 2. For KE there is one outlier: subject 6, who has the lowest KE in stress. Also for EL there is one outlier: subject 1, who has the highest EL in stress. 
Table 1 Characteristics of the study group

\begin{tabular}{|c|c|c|c|c|c|}
\hline & Rest & Dobutamine stress & Difference & Difference (\%) & $P$-value \\
\hline & Mean $\pm S D$ & Mean \pm SD & $\begin{array}{l}\text { Stress-Rest } \\
\text { Mean } \pm \text { SD or Median [IQR] }\end{array}$ & $\begin{array}{l}(\text { Stress-rest)/rest } \times 100 \% \\
\text { Mean } \pm \text { SD or Median }[\mathrm{IQR}]\end{array}$ & \\
\hline Characteristics of the study group & at underwent & whole protocol $(n=$ & & & \\
\hline Age (years) & $16.5 \pm 3.8$ & $\mathrm{n} / \mathrm{a}$ & $\mathrm{n} / \mathrm{a}$ & $\mathrm{n} / \mathrm{a}$ & $\mathrm{n} / \mathrm{a}$ \\
\hline Male (\%) & $4 / 10(40)$ & $\mathrm{n} / \mathrm{a}$ & $\mathrm{n} / \mathrm{a}$ & $\mathrm{n} / \mathrm{a}$ & $\mathrm{n} / \mathrm{a}$ \\
\hline $\mathrm{VO}_{2} \max ^{\mathrm{a}}$ & $30.2 \pm 8.6$ & $\mathrm{n} / \mathrm{a}$ & $\mathrm{n} / \mathrm{a}$ & $\mathrm{n} / \mathrm{a}$ & $\mathrm{n} / \mathrm{a}$ \\
\hline Oxygen saturation ${ }^{a}$ & $93.8 \pm 4.4^{\mathrm{b}}$ & $88.7 \pm 4.8^{\mathrm{b}}$ & $-4.3 \pm 3.3$ & $-5 \pm 4$ & 0.008 \\
\hline HR (bpm) & $83.4 \pm 18.9$ & $110.9 \pm 20.0$ & $27.5 \pm 8.2$ & $35 \pm 12$ & $<0.001$ \\
\hline EDV $(\mathrm{mL})$ & $164.4 \pm 43.9$ & $156.8 \pm 46.0$ & $-7.6 \pm 13.9$ & $-5 \pm 8$ & 0.12 \\
\hline ESV (mL) & $83.6 \pm 23.7$ & $64.8 \pm 20.9$ & $-21.7[-25.6$ to -14.4$]$ & $-26[-30$ to -19$]$ & $<0.001$ \\
\hline$S V(m L)$ & $80.8 \pm 29.4$ & $92.0 \pm 33.9$ & $11.1 \pm 9.2$ & $14 \pm 10$ & 0.004 \\
\hline EF (\%) & $49.1 \pm 8.5$ & $58.6 \pm 8.1$ & $9.5 \pm 3.2$ & $20 \pm 8$ & $<0.001$ \\
\hline $\mathrm{CO}(\mathrm{L} / \mathrm{min})$ & $6.4 \pm 1.3$ & $9.7 \pm 2.2$ & $3.3 \pm 1.3$ & $52 \pm 17$ & $<0.001$ \\
\hline Systolic blood pressure $(\mathrm{mmHg})$ & $121.2 \pm 15.2$ & $150.6 \pm 19.3$ & $29.4 \pm 12.1$ & $25 \pm 11$ & $<0.001$ \\
\hline Diastolic blood pressure $(\mathrm{mmHg})$ & $62.7 \pm 10.0$ & $70.3 \pm 6.0$ & $7.6 \pm 9.5$ & $14 \pm 19$ & 0.03 \\
\hline Sphericity index systole & $1.1 \pm 0.3$ & $1.2 \pm 0.4$ & $0.1 \pm 0.2$ & $2[-8$ to 13$]$ & 0.44 \\
\hline Sphericity index diastole & $1.0 \pm 0.1$ & $1.1 \pm 0.2$ & $0.05 \pm 0.2$ & $2[-5$ tot 9$]$ & 0.41 \\
\hline
\end{tabular}

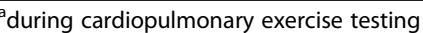

boxygen saturation was missing for one patient

$H R$ heart rate, EDV end diastolic volume, ESV end systolic volume, SV stroke volume, CO cardiac output, EF ejection fraction, VO2 max maximal oxygen uptake IQR interquartile range

Results of KE, EL and vorticity_vol at rest and dobutamine stress normalized by SV are also shown in Table 2. Normalized by stroke volume, KE and EL values still showed a significant increase over the total cycle, similar to the non-normalized values.

\section{Association between difference HR, stroke volume and blood pressure versus $K E$, EL and vorticity}

The difference in HR between rest and stress was not associated with the difference in KE, EL or vorticity vol $\left(\mathrm{KE}_{\text {avg cycle }}: r=-0.27, P=0.45\right.$; $\mathrm{EL}_{\text {avg cycle: }} r=0.01$,

Table 2 Quantitative analysis of energetics and vorticity at rest and stress

\begin{tabular}{|c|c|c|c|c|}
\hline & Rest & Dobutamine stress & Difference(\%) & $P$-value \\
\hline & Mean \pm SD or Median [IQR] & Mean \pm SD & $\begin{array}{l}(\text { Stress-rest)/rest } \times 100 \% \\
\text { Mean } \pm \text { SD or Median }[\mathrm{IQR}]\end{array}$ & \\
\hline \multicolumn{5}{|l|}{ Non-normalized } \\
\hline $\mathrm{KE}_{\text {avg systole }}(\mathrm{mJ})$ & $2.4 \pm 1.1$ & $5.1 \pm 1.8$ & 99 [72-195] & $<0.001$ \\
\hline $\mathrm{KE}_{\text {avg diastole }}(\mathrm{mJ})$ & $1.4 \pm 0.3$ & $2.2 \pm 0.7$ & $61 \pm 57$ & 0.007 \\
\hline $\mathrm{KE}_{\text {avg cycle }}(\mathrm{mJ})$ & $1.8 \pm 0.5$ & $3.3 \pm 0.9$ & $88 \pm 52$ & $<0.001$ \\
\hline $\mathrm{EL}_{\text {avg systole }}(\mathrm{mW})$ & $1.2[0.6-1.5]$ & $3.1 \pm 1.8$ & $155 \pm 61$ & 0.005 \\
\hline $\mathrm{EL}_{\text {avg diastole }}(\mathrm{mW})$ & $0.7 \pm 0.2$ & $1.2 \pm 0.5$ & $71 \pm 66$ & 0.007 \\
\hline $\mathrm{EL}_{\text {avg cycle }}(\mathrm{mW})$ & $0.9 \pm 0.4$ & $1.9 \pm 0.9$ & $108 \pm 49$ & $<0.001$ \\
\hline Vorticity_vol avg systole $(\mathrm{mL} / \mathrm{s})$ & $3592 \pm 1148$ & $4928 \pm 1626$ & $37 \pm 17$ & $<0.001$ \\
\hline Vorticity_vol $_{\text {avg diastole }}(\mathrm{mL} / \mathrm{s})$ & $3371 \pm 770$ & $4086 \pm 1216$ & $21 \pm 25$ & 0.02 \\
\hline Vorticity_vol avg cycle $_{\text {(mL/s) }}$ & $3441 \pm 899$ & $4394 \pm 1322$ & $27 \pm 19$ & 0.002 \\
\hline \multicolumn{5}{|l|}{ Normalized by stroke volume } \\
\hline $\mathrm{KE}_{\text {avg cycle }} / \mathrm{SV}(\mathrm{mJ} / \mathrm{mL})$ & $0.023 \pm 0.005$ & $0.037 \pm 0.009$ & $64 \pm 40$ & $<0.001$ \\
\hline $\mathrm{EL}_{\text {avg cycle }} / \mathrm{SV}(\mathrm{mW} / \mathrm{mL})$ & $0.012 \pm 0.004$ & $0.021 \pm 0.009$ & $81 \pm 36$ & $<0.001$ \\
\hline Vorticity_vol $_{\text {avg cycle/SV (1/s) }}$ & $44.1 \pm 9.7$ & $49.1 \pm 11.1$ & $11 \pm 11$ & 0.006 \\
\hline
\end{tabular}

$S D$ standard deviation, $K E$ kinetic energy, SV stroke volume, EL viscous energy loss IQR interquartile range 

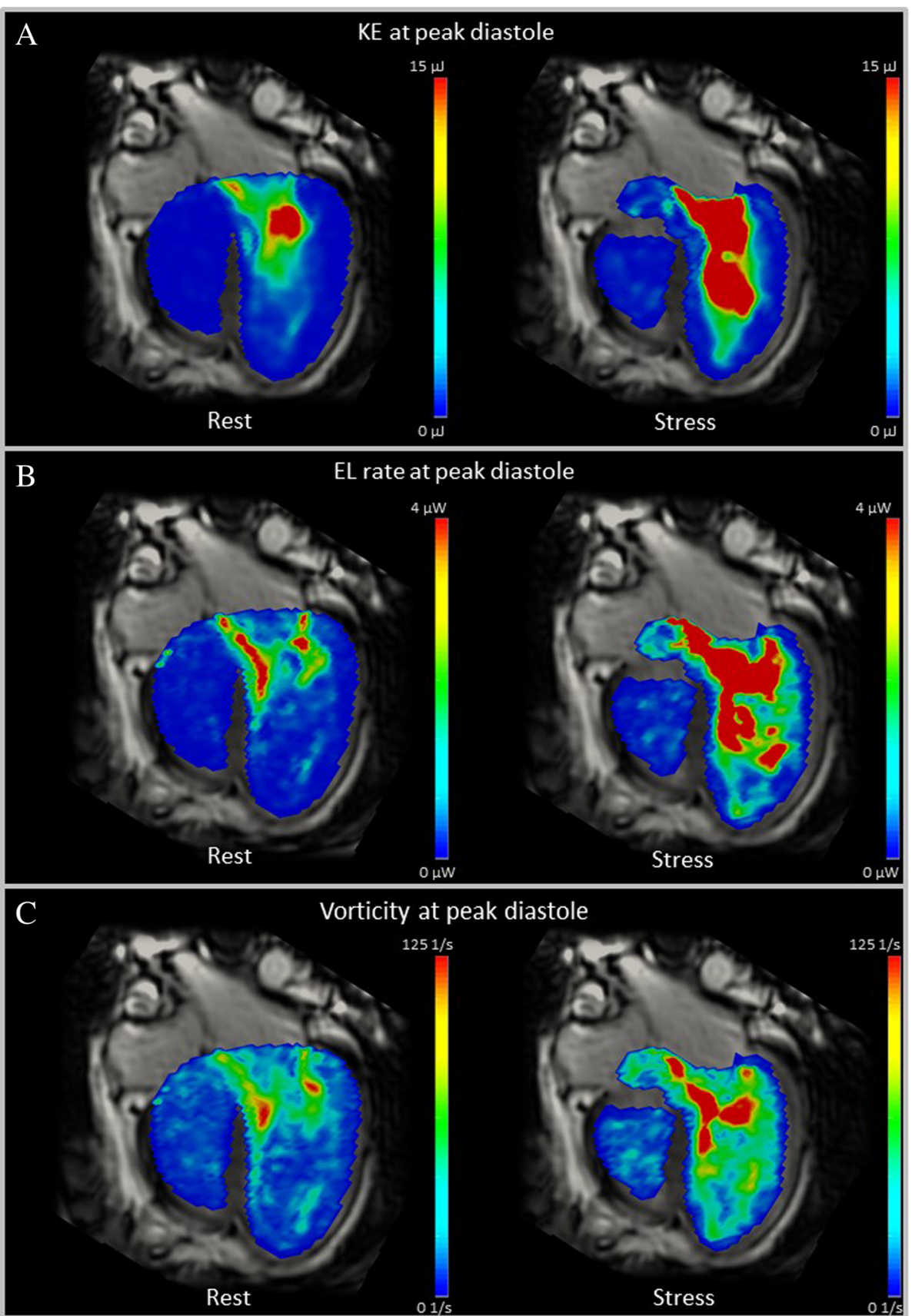

Fig. 1 Intracardiac hemodynamics at rest and dobutamine stress in a patient with pulmonary atresia at peak diastolic filling. a shows the intraventricular kinetic energy (KE) at rest and stress. b shows the intraventricular viscous energy loss (EL) rate at rest and stress. c shows the intraventricular vorticity at rest and stress

$P=0.97$; vorticity_vol $\left.{ }_{\text {avg cycle: }} r=-0.26, P=0.48\right)$. The increase in SV (from 2D planimetry) between rest and stress was significantly associated with the difference in vorticity_vol $_{\text {avg cycle }}$ (vorticity_vol ${ }_{\text {avg cycle: }} 0.72, P=0.02$ ), but not with the difference in KE or EL (KE $\mathrm{Kevg}_{\text {cycle: }} r=-0.46$, $P=0.19$; $\mathrm{EL}_{\text {avg cycle }}: r=0.48, P=0.16$ ). There was no correlation between the absolute change in diastolic blood pressure from CMR and the absolute change in diastolic energy parameters (KE: $r=-0.11, P=0.77$ and EL: $r=0.12, P=$ $0.73)$, or the percentage change in diastolic blood pressure from CMR and percentage change in diastolic energy parameters (KE: $r=0.08, P=0.83$ and EL: $r=0.02, P=0.96$ ). Also, there was no correlation between the absolute change in systolic blood pressure from CMR and absolute change in systolic energy parameters (KE: $r=-0.31, P=0.38$ and EL: $r=0.43, P=0.21)$. There was a moderate correlation 

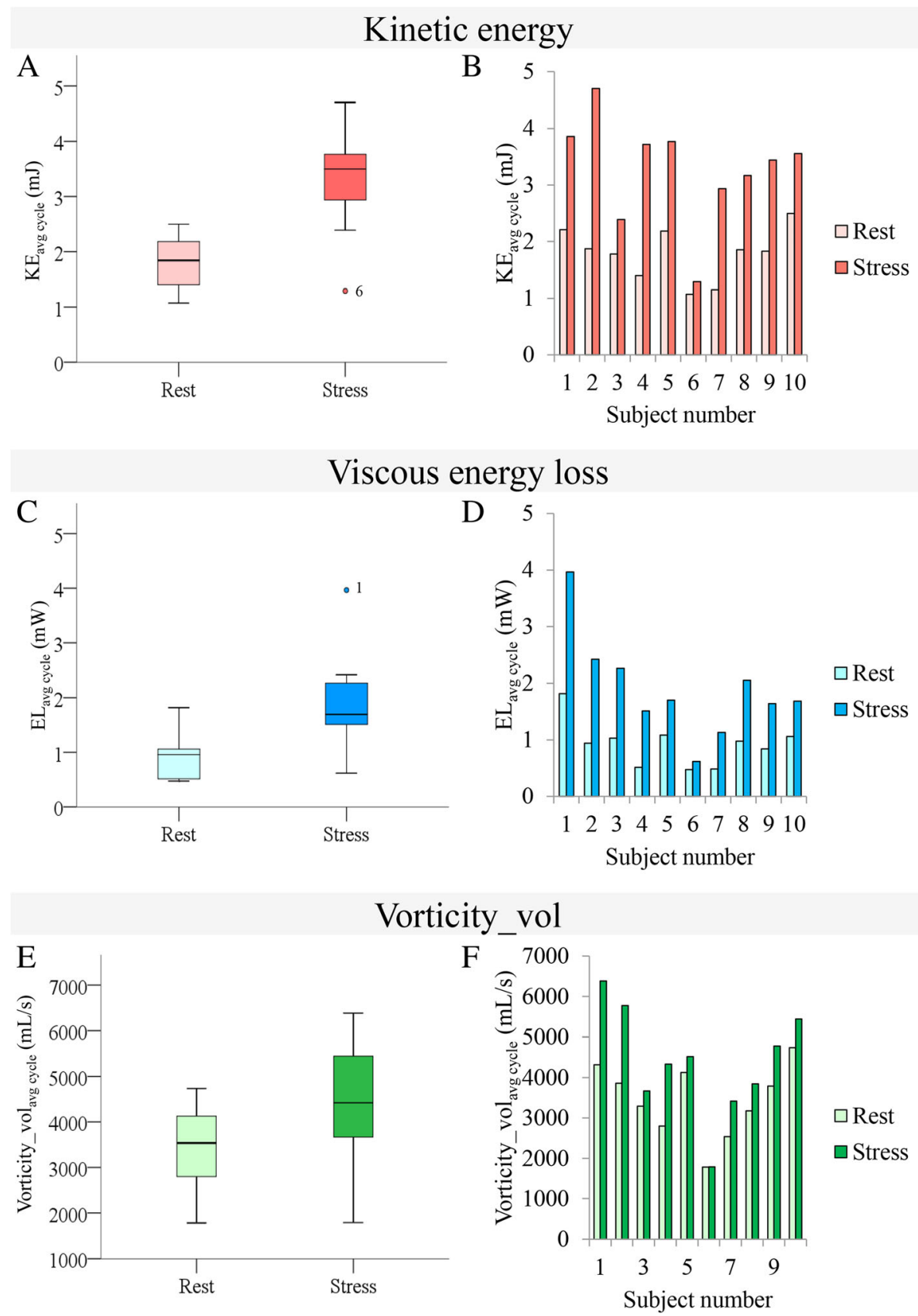

Fig. 2 Relative difference in intraventricular kinetic energy (KE), viscous energy loss (EL) and vorticity (vorticity_vol) over the total cardiac cycle between rest and stress. a Difference in KE in the total group in rest and stress. There is one outlier: subject 6 , who has the lowest KE in stress; $\mathbf{b}$ Difference in KE per subjects in rest and stress. There is one outlier: subject 1, who has the highest EL in stress; $\mathbf{c}$ Difference in EL in the total group in rest and stress; $\mathbf{d}$ Difference in EL per subjects in rest and stress; e Difference in vorticity in the total group in rest and stress; $\mathbf{f}$ Difference in vorticity per subjects in rest and stress. The scales are per voxel -lowest voxel value would be 0 and maximum would be the highest on the corresponding scale

between the percentage change in systolic blood pressure from CMR and the percentage change in systolic KE $(r=-$ $0.67, P=0.04)$, but not in EL $(r=-0.35, P=0.32)$.

\section{Relation to VO2 max from cardiopulmonary exercise testing}

Figure 3 shows the scatter plot between $\mathrm{VO}_{2}$ max from $\mathrm{CPET}$ and the relative rest-stress difference in $\mathrm{KE}, \mathrm{EL}$ and vorticity. This plot shows a significant good inverse correlation between $\mathrm{VO}_{2}$ max from CPET and relative $\mathrm{KE}_{\text {avg cycle }}$ rest-stress difference $(r=-0.83, P=0.003)$. Furthermore, there is a significant good inverse correlation between $\mathrm{VO}_{2}$ max from CPET and relative $\mathrm{EL}_{\text {avg }}$ cycle rest-stress difference $(r=-0.80, P=0.006)$. Also, $\mathrm{VO}_{2}$ max from CPET and relative vorticity_vol ${ }_{\text {avg }}$ cycle 


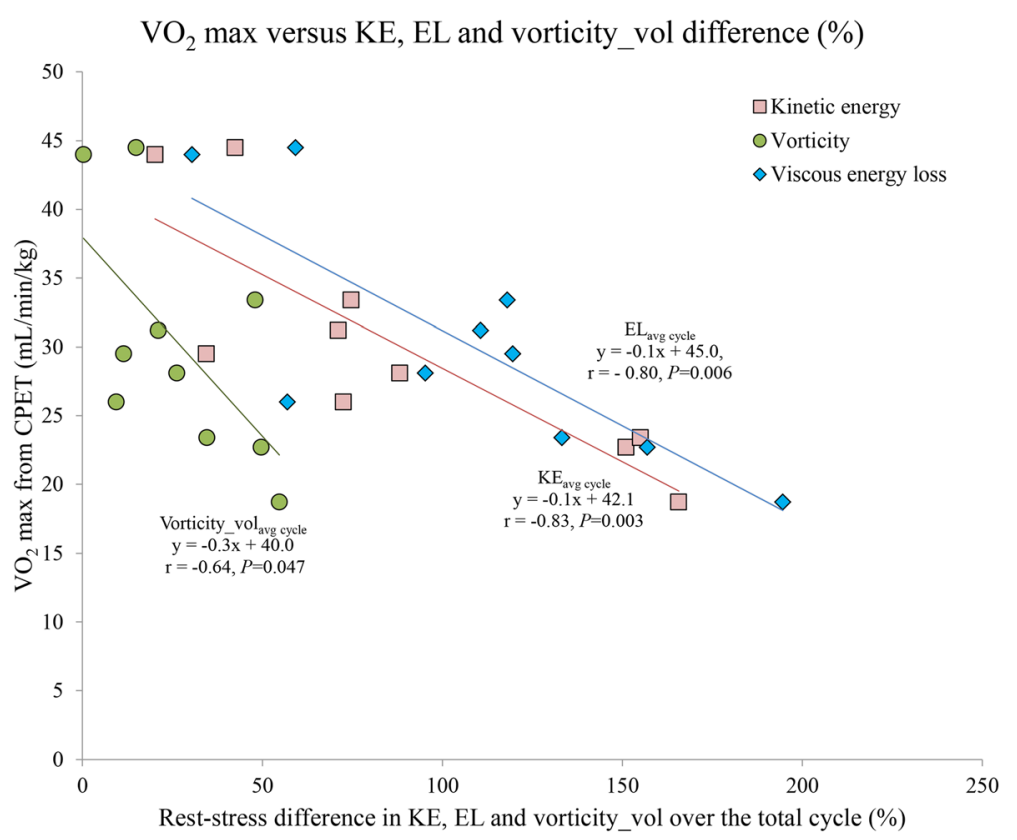

Fig. 3 Scatter plot showing the relation between maximal oxygen uptake $\left(\mathrm{VO}_{2}\right.$ max) from cardiopulmonary exercise tests (CPET) and the relative difference in intraventricular kinetic energy (KE), viscous energy loss (EL) and vorticity (vorticity_vol) over the total cardiac cycle between rest and stress

rest-stress difference show a significant moderate inverse correlation $(r=-0.64, P=0.047)$. In the two patients with normal $\mathrm{VO}_{2}$ max [19] the relative rest-stress difference in vorticity_vol was $0.3-15 \%$, the relative rest-stress difference in KE $20-42 \%$ and in EL $30-59 \%$. The patient with the lowest $\mathrm{VO}_{2}$ max showed a $55 \%$ increase in vorticity_vol, but a $166 \%$ increase in KE and a $195 \%$ increase in EL during stress. Overall, decreased $\mathrm{VO}_{2}$ max is related to increases in vorticity_vol, KE and EL reststress differences, though both relative rest-stress differences in KE and EL showed a three-fold stronger increase than relative difference in vorticity_vol.

\section{Discussion}

In the current study, the effect of pharmacologic stress on intraventricular KE, EL and vorticity, derived from 4D flow CMR was evaluated in Fontan patients and the relation was tested between stress response on these markers and exercise capacity, measured by $\mathrm{VO}_{2} \max$ from cardiopulmonary exercise testing. Main findings of the study are: 1) intraventricular KE, EL and vorticity vol increased during pharmacologic stress; 2) the increase in $\mathrm{KE}$ and EL under stress was 3-4 times higher than the increase in vorticity_vol; 3) $\mathrm{VO}_{2} \max$ from CPET showed a significant negative correlation with the relative rest-stress differences in KE, EL and vorticity vol. 4) at identical $\mathrm{VO}_{2}$ max value, $\mathrm{KE}$ and EL showed a three-fold higher increase between rest and stress compared to relative rest-stress difference in vorticity_vol. In the normal left ventricle at rest, blood flow follows the path that is most efficient for optimal ejection of flow in the systemic circulation. The shape of the healthy left ventricle and normal mitral valve contributes to the formation of a recirculating flow pattern during diastole, forming a confined region of vortical flow that conserves momentum by storing KE, while during systole the flow follows a rather semi-circular path from the mitral valve towards the left ventricular outflow tract $[14,15]$. During stress the complex dynamic flow patterns in the heart might change in order to achieve redirection of momentum through the curved paths of flow [26]. An in vivo study in the hearts of healthy pigs [27] showed increased 4D flow CMR-derived trans-mitral diastolic inflow velocity and unfavorable conditions for diastolic vortex development under dobutamine stress. However, the effect of pharmacologic stress on 4D flow CMR-derived intraventricular flow patterns and $\mathrm{KE}, \mathrm{EL}$ and vorticity in the human healthy left ventricle, has not been published.

Fontan patients have various structural ventricular and valvular anomalies. Moreover, the ventricle that sustains the systemic circulation can be of left ventricular or right ventricular morphology. Furthermore, in some patients, diastolic inflow occurs in the same ventricle as from which blood is ejected during systole (i.e., as is the case in a patient with tricuspid atresia with a normal ventriculo-arterial connection) while in other patients, inflowing blood has to pass through a ventricular septal defect first to reach the aorta for ejection (i.e., as is the case in patients with double inlet left ventricle with the aorta arising from a hypoplastic right ventricle). The abnormal 
intracardiac anatomy in Fontan patients causes differences in intraventricular flow patterns, which can be assessed and studied by 4D flow CMR [17]. In a similar way, altered flow patterns have been visualized and quantified in patients with a corrected atrioventricular septal defect [28] in which altered vortex formation was associated with increased EL obtained by $4 \mathrm{D}$ flow CMR [21]. Furthermore, in a patient with a Fontan circulation with a complete atrioventricular septal defect and a double outlet right ventricle with pulmonary stenosis, abnormal intracardiac structures have been linked to regions of vortex formation and increased EL [16]. In Fontan patients, decreased diastolic KE was reported versus healthy controls [18], as well as increased EL [22]. However, all these published studies focused on patients at rest and the response in intracardiac 4D flow CMR-derived hemodynamic parameters such as KE, EL and vorticity during stress has not been evaluated earlier.

Currently 4D flow CMR is challenging during physical exercise due to the incompatibility of the long scans times needed for 4D flow imaging and the movements inherent to physical exercise within the CMR scanner. Our study shows that pharmacologic (dobutamine-induced) stress results in a significant increase in intracardiac KE, EL and vorticity_vol from 4D flow CMR. The percentage increase was different between all individual subjects (Fig. 2, Table 3 in Appendix 2), which is dependent on ventricular size, the systemic ventricle, and could also be dependent on whether the patient has an extracardiac conduit or a lateral tunnel. The sample size is too small to permit more detailed analysis but it is clear that the only patient with a lateral tunnel (subject 5) has a different response to stress compared to a different patient with similar underlying anatomy but with a extracardiac conduit (subject 6 ). We could speculate that this may be explained by preload dependency i.e. it could mean that the lateral tunnel (which is an intra-atrial tunnel) inhibits preload under stress.

Notably, in the current study the dobutamine stressinduced increase in KE and EL is 3-4 times higher than the increase in vorticity_vol $\left(\mathrm{KE}_{\text {avg }}\right.$ cycle increase $88 \%$; $\mathrm{EL}_{\text {avg cycle }}$ increase $108 \%$, vorticity_vol $\mathrm{avg}_{\text {cycle }}$ increase 27\%). The difference in the increase in KE and EL compared to vorticity_vol could partly be explained by the abnormal underlying anatomy of the Fontan patients. Increased KE results in higher EL, which is possibly enhanced by more flow-structure interaction due to abnormal anatomy. However, the increase in vorticity vol is not in the same order of magnitude. Vorticity in the ventricular flow might have a favorable effect (i.e., conservation of momentum by KE storage and redirection of flow during diastole which takes place in a confined region of vortical flow distal to the mitral valve) $[14,15]$. However, vorticity may also have an adverse effect on the intracardiac blood flow when optimal flow redirection may be perturbed by flow separation and vortex shedding distal to abnormal intracardiac structures in the flow field [29-31]. In this study, we are not able to discriminate between favorable and adverse effects of vorticity. In either case, the KE contributes to vortex formation but this study showed that stress results in a higher increase in KE than in vorticity. The relation between vortex formation and flow velocity (i.e. kinetic energy) has been studied before: Gharib et al. [29] described that the volume and circulation of a vortex ring that is formed from inflow through a circular nozzle into a large unconfined volume would both increase with increasing inflow velocity, but only up to a critical point where optimal vortex formation has been achieved. At this optimal point, both volume and vorticity of the main vortex will remain approximately constant. However, secondary vortices may start to appear that will contribute to the total summed vorticity. Furthermore, in our study the shape of the ventricle measured by the sphericity index and end-diastolic volume remained similar during rest and stress. This could support the hypothesis that the size of the vortical flow region during diastole is restricted in growth and unable to expand at the same rate as the KE increase during stress.

Survival after the Fontan procedure has increased drastically over the past decades [2, 3]. Still, patients with a Fontan circulation exhibit diminished exercise capacity, related to worse functional health status [4-6]. Several factors have been identified that play a role in the reduced exercise capacity in these patients [7-11]. However, the only previous study that related exercise capacity to EL was aimed at the TCPC of Fontan patients [10] and not at intraventricular flow dynamics. In the current study, an increase in the relative rest-stress difference in KE, EL and vorticity_vol was inversely correlated with $\mathrm{VO}_{2}$ max, though notably relative rest-stress differences in KE and EL showed a three-fold stronger increase than relative difference in vorticity_vol. This is in line with our previous speculation that, in patients with lower exercise capacity, storage capacity of KE inside the confined vortical flow region is limited and therefore, does not proportionally follow the increase in total KE. Furthermore, abnormal intraventricular anatomy could play a role in this relation as well. Nevertheless, our findings may provide evidence that intracardiac hemodynamics and energetics play a role in exercise capacity in Fontan patients.

Our results showed no association between the difference in HR and the difference in KE, EL or vorticity. However, the HR increase during the low-dose dobutamine stress protocol we used is limited to a $30 \%$ increase. From this study, we cannot determine whether this relation would be similar when HR increase is higher. Also, we showed that SV difference is directly 
related to vorticity difference, but not to the difference in KE or EL.

In the current study we evaluated intracardiac 4D flow hemodynamic parameters in a rest -stress setting and associated the results with an exercise parameter $\left(\mathrm{VO}_{2} \max \right)$. In Fontan patients, there is a delicate interplay between preload and afterload and efficiency of the TCPC and aortic outflow is also of great importance. This study is a first step in the evaluation of the intricate hemodynamic status of the Fontan patient in exercise. Future studies should also relate TCPC hemodynamics during stress with intracardiac hemodynamics during stress.

\section{Limitations}

This study has a number of limitations. One limitation is the lack of a control group in order to compare these results in Fontan patients to a normal range. However, studying the effect of pharmacological (dobutamine) stress on 4D flow CMR-derived intraventricular flow patterns and KE, EL and vorticity in the healthy left ventricle in children requires a strict and thorough ethical consideration and the current study was not eligible for such test. Another limitation of the current study is the small sample size. However, our results are still highly statistically significant. Because of the small sample size, we could not evaluate individual differences between patients with separate underlying anatomies. Furthermore, we did not include an evaluation of turbulent kinetic energy, which could be relevant in Fontan patients, as turbulent flow may be present due to the structural intraventricular abnormalities. Additionally, 4D flow CMR is a time-resolved imaging modality that only represents time-averaged blood flow acquired over multiple heart cycles. Consequently, this limits the evaluation to coherent large-scale (within 4D flow CMR resolution) vortical flow only, while incoherent unstable or small-scale flow, especially during a stress-induced increase in cardiac workload, could not be measured, although the effect may not be negligible. We did not evaluate any changes in afterload induced by stress and therefore the influence of changes in afterload on ventricular energetics, which could be subject for further research. Lastly, in this study the 4D flow CMR results under pharmacologic stress were compared to $\mathrm{VO}_{2}$ max during exercise stress. There could be a potential confounding effects of 4D flow CMR measurements performed while supine versus exercise measurements performed upright [32], especially in Fontan patients [33]. It would have been theoretically ideal to perform the exercise testing with a supine ergometer to minimize this confounder, however this was not possible in our study protocol. Further studies should evaluate this effect.

\section{Clinical perspectives}

This study provides evidence that intracardiac vortical flow and energetics play a role in exercise capacity in Fon$\tan$ patients. Although still speculative, these results could potentially influence patient follow-up and surgical planning. Insight in hemodynamic parameters during stress may provide a quantitative index that could help better understand how the mechanism of which underlying pathophysiology effects the response to stress and the influence on exercise capacity. However, larger future studies are needed to confirm the results of this pilot study.

\section{Conclusions}

In conclusion, intraventricular kinetic energy, viscous energy loss and vorticity values derived from 4D flow CMR increased during pharmacologic stress, with a three to four times higher increase in KE and EL compared to the vorticity increase. The increase in KE, EL and vorticity showed a negative correlation with exercise capacity measured by $\mathrm{VO}_{2}$ max from cardiopulmonary exercise testing. At identical $\mathrm{VO}_{2}$ max value, both relative rest-stress differences in $\mathrm{KE}$ and EL showed a three-fold stronger increase than the relative differences in vorticity.

\section{Appendix 1 \\ CMR acquisition information}

A complete CMR scan including whole-heart 4D flow CMR was obtained on a $3 \mathrm{~T}$ scanner (Ingenia, Philips Healthcare, Best, the Netherlands) with maximal amplitude of $45 \mathrm{mT} / \mathrm{m}$ for each axis and slew rate of $200 \mathrm{~T} /$ $\mathrm{m} / \mathrm{s}$. A combination of FlexCoverage Posterior coil in the table top with a dStream Torso coil, providing up to 32 coil elements was used for signal reception. Velocityencoding of $150 \mathrm{~cm} / \mathrm{s}$ in all three directions was used in a standard four-point encoding scheme, spatial resolution $3.0 \times 3.0 \times 3.0 \mathrm{~mm}^{3}$ or better, flip angle $10^{\circ}$, echo time (TE) $3.7 \mathrm{~ms}$, repetition time (TR) $7.7-10 \mathrm{~ms}$, true temporal resolution $30-40 \mathrm{~ms}$, sensitivity encoding factor 2 in anterior-posterior direction and echo planar imaging readout with a factor 5. Concomitant gradient correction and phase offset correction was performed using standard available scanner software. Typical acquisition time of the whole-heart 4D flow CMR scan was approximately $8 \mathrm{~min}$. Cine two-dimensional left 2-chamber, 4-chamber, coronal and sagittal aorta views and transversal images were acquired, using balanced steadystate free-precession sequences with TE/TR 1.5/3.0, 350 $\mathrm{mm}$ field-of-view, $45^{\circ}$ flip angle, acquisition resolution $1.9 \times 2.0 \times 8.0 \mathrm{~mm}^{3}$. Retrospective gating was used with 30 phases reconstructed to represent one cardiac cycle. Free breathing was allowed without using motion suppression; three signal averages were taken to minimize effects of breathing motion. 


\section{Appendix 2}

Table 3 Subject specific parameters

\begin{tabular}{|c|c|c|c|c|c|c|c|c|}
\hline \multicolumn{9}{|c|}{ Subject specific parameters } \\
\hline \multicolumn{2}{|c|}{ Subject Underlying anatomy } & $\begin{array}{l}\text { Ventricular } \\
\text { morphology }\end{array}$ & $\begin{array}{l}\text { Type of } \\
\text { Fontan }\end{array}$ & VO2max ${ }^{a}$ & $\begin{array}{l}\text { Rest-stress diff } \\
\text { EDV (\%) }\end{array}$ & $\begin{array}{l}\text { Rest-stress diff } \\
\mathrm{KE}_{\text {avg cycle }}(\%)\end{array}$ & 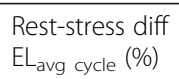 & $\begin{array}{l}\text { Rest-stress diff } \\
\text { Vorticity_volavg cycle (\%) }\end{array}$ \\
\hline 1 & $\begin{array}{l}\text { Unbalanced AVSD, } \\
\text { hypoplastic LV }\end{array}$ & Biventricular & Extracardiac & 33.4 & 13.07 & 74.64 & 58.17 & 47.98 \\
\hline 2 & TGA,VSD, PS, hypoplastic LV & Biventricular & Extracardiac & 22.7 & .71 & 151.04 & 93.35 & 49.66 \\
\hline 3 & $\begin{array}{l}\text { cCTGA, VSD, PS, straddling } \\
\text { TV }\end{array}$ & Biventricular & Extracardiac & 29.5 & -12.97 & 34.45 & 77.63 & 11.44 \\
\hline 4 & PA & Left & Extracardiac & 18.7 & -2.54 & 165.67 & 39.92 & 54.71 \\
\hline 5 & TA & Left & $\begin{array}{l}\text { Lateral } \\
\text { tunnel }\end{array}$ & 26.0 & -16.70 & 72.51 & 8.07 & 9.40 \\
\hline 6 & TA & Left & Extracardiac & 44.0 & -8.00 & 20.18 & -3.54 & .31 \\
\hline 7 & PA & Left & Extracardiac & 23.4 & -9.23 & 155.04 & 80.78 & 34.58 \\
\hline 8 & DILV, with aorta from RV & Left & Extracardiac & 31.2 & -10.15 & 71.06 & 40.02 & 21.10 \\
\hline 9 & HLHS & Right & Extracardiac & 28.1 & -2.88 & 88.17 & 71.19 & 26.23 \\
\hline 10 & TGA,VSD, PS, hypoplastic LV & Right & Extracardiac & 44.5 & -1.35 & 42.44 & 7.91 & 14.96 \\
\hline
\end{tabular}

afrom cardiopulmonary exercise testing

EDV end diastolic volume, VO2 max maximal oxygen uptake, AVSD atrioventricular septal defect, LV left ventricle, TGA transposition of the great arteries, VSD ventricular septal defect, PS pulmonary stenosis, $T V$ tricuspid valve, $P A$ pulmonary atresia, $T A$ tricuspid atresia, DILV double inlet right ventricle, $R V$ right ventricle, HLHS hypoplastic left heart syndrome

\section{Abbreviations}

4D: Four-dimensional; Cl: Confidence interval; CMR: Cardiovascular magnetic resonance; CO: Cardiac output; CPET: Cardiopulmonary exercise testing; EDV: End-diastolic volume; EF: Ejection fraction; EL: Viscous energy loss; ESV: End-systolic volume; HR: Heart rate; IQR: Interquartile range; KE: Kinetic energy; LV: Left ventricle/left ventricular; SV: Stroke volume; TCPC: Total cavopulmonary connection; $\mathrm{VO}_{2}$ max: Maximum oxygen uptake; vorticity_vol: Vorticity integral over the ventricular volume

\section{Acknowledgements}

\section{Not applicable.}

\section{Authors' contributions}

All authors made appropriate contributions to the manuscript. All authors were involved in the study design. VPK, MSME, AAWR and JJMW drafted the manuscript. MSME developed the algorithms and software for vorticity, EL and KE computation from 4D Flow CMR. VPK, PJvdB and JJMW participated in the data acquisition. VPK, LJMK, JJMW, AAWR and MSME were involved in the data analysis. VPK performed the statistical analysis. All authors critically reviewed and approved the final manuscript.

\section{Funding}

V.P. Kamphuis is financially supported by a grant from the Dutch Heart Foundation (Grant Number 2013 T091). J.J.M. Westenberg is financially supported by a grant of ZonMw (project number 104003001).

\section{Availability of data and materials}

The datasets used and/or analysed during the current study are available from the corresponding author on reasonable request.

\section{Ethics approval and consent to participate}

All patients were prospectively evaluated in this study at the Leiden University Medical Center (LUMC) as part of a multicenter study that was approved by the Medical Ethical Committee of the Erasmus Medical Center in Rotterdam (MEC2014-326, NL48188.078.14), with local approval of the Medical Ethical Committee of the LUMC, The Netherlands. Informed consent was obtained from all of these participants. All methods were performed in accordance with the relevant guidelines and regulations.

\section{Consent for publication}

Not applicable

\section{Competing interests}

The authors declare that they have no competing interests.

\section{Author details}

'Department of Pediatrics division of Pediatric Cardiology, Leiden University Medical Center, Leiden, the Netherlands. ${ }^{2}$ Netherlands Heart Institute, Utrecht, The Netherlands. ${ }^{3}$ Department of Radiology Feinberg School of Medicine, Northwestern University, Chicago, USA. ${ }^{4}$ Department of Radiology, Leiden University Medical Center, Leiden, the Netherlands. ${ }^{5}$ Department of Cardiothoracic Surgery, Leiden University Medical Center, Leiden, the Netherlands. ${ }^{6}$ Department of Cardiology, Leiden University Medical Center, Leiden, the Netherlands. ${ }^{7}$ Department of Pediatrics division of Pediatric Cardiology, Academic Medical Center, Amsterdam, the Netherlands. ${ }^{8}$ Department of Pediatrics, division of Pediatric Cardiology, Erasmus Medical Center, Rotterdam, the Netherlands. ${ }^{9}$ Department of Pediatrics division of Pediatric Cardiology, Radboud university Medical Center, Nijmegen, the Netherlands.

Received: 18 January 2019 Accepted: 14 June 2019

Published online: 25 July 2019

\section{References}

1. Fontan F, Baudet E. Surgical repair of tricuspid atresia. Thorax. 1971; 26(3):240-8.

2. Chin AJ, Whitehead KK, Watrous RL. Insights after 40 years of the fontan operation. World journal for pediatric \& congenital heart surgery. 2010;1(3): 328-43

3. Gersony WM. Fontan operation after 3 decades: what we have learned. Circulation. 2008;117(1):13-5.

4. Atz AM, Zak V, Mahony L, Uzark K, D'Agincourt N, Goldberg DJ, et al. Longitudinal outcomes of patients with single ventricle after the Fontan procedure. J Am Coll Cardiol. 2017;69(22):2735-44.

5. Hock J, Reiner B, Neidenbach RC, Oberhoffer R, Hager A, Ewert P, et al. Functional outcome in contemporary children with total cavopulmonary connection - health-related physical fitness, exercise capacity and healthrelated quality of life. Int J Cardiol. 2018;255:50-4.

6. Robbers-Visser D, Kapusta L, van Osch-Gevers L, Strengers JL, Boersma E, de Rijke YB, et al. Clinical outcome 5 to 18 years after the Fontan operation 
performed on children younger than 5 years. J Thorac Cardiovasc Surg. 2009;138(1):89-95.

7. Bossers SS, Helbing WA, Duppen N, Kuipers IM, Schokking M, Hazekamp MG, et al. Exercise capacity in children after total cavopulmonary connection: lateral tunnel versus extracardiac conduit technique. J Thorac Cardiovasc Surg. 2014;148(4):1490-7.

8. Bossers SS, Kapusta L, Kuipers IM, van Iperen G, Moelker A, Kroft $L$, et al. Ventricular function and cardiac reserve in contemporary Fontan patients. Int J Cardiol. 2015;196:73-80.

9. Greutmann M, Le TL, Tobler D, Biaggi P, Oechslin EN, Silversides CK, et al. Generalised muscle weakness in young adults with congenital heart disease. Heart. 2011;97(14):1164-8.

10. Khiabani RH, Whitehead KK, Han D, Restrepo M, Tang E, Bethel J, et al. Exercise capacity in single-ventricle patients after Fontan correlates with haemodynamic energy loss in TCPC. Heart. 2015;101(2):139-43.

11. Matthews IL, Fredriksen PM, Bjornstad PG, Thaulow E, Gronn M. Reduced pulmonary function in children with the Fontan circulation affects their exercise capacity. Cardiol Young. 2006;16(3):261-7.

12. Tang E, Wei ZA, Whitehead KK, Khiabani RH, Restrepo M, Mirabella L, et al. Effect of Fontan geometry on exercise haemodynamics and its potential implications. Heart. 2017;103(22):1806-12.

13. Kamphuis VP, Westenberg JJM, van der Palen RLF, Blom NA, de Roos A, van der Geest R, et al. Unravelling cardiovascular disease using four dimensional flow cardiovascular magnetic resonance. Int J Cardiovasc Imaging. 2017; 33(7):1069-81.

14. Kilner PJ, Yang GZ, Wilkes AJ, Mohiaddin RH, Firmin DN, Yacoub MH. Asymmetric redirection of flow through the heart. Nature. 2000;404(6779):759-61.

15. Pedrizzetti G, Domenichini F. Nature optimizes the swirling flow in the human left ventricle. Phys Rev Lett. 2005;95(10):108101.

16. Kamphuis VP, Roest AAW, Westenberg JJM, Elbaz MSM. Biventricular vortex ring formation corresponds to regions of highest intraventricular viscous energy loss in a Fontan patient: analysis by 4D flow MRI. Int J Cardiovasc Imaging. 2018a;34(3):441-2.

17. She HL, Roest AA, Calkoen EE, van den Boogaard PJ, van der Geest RJ, Hazekamp MG, et al. Comparative evaluation of flow quantification across the atrioventricular valve in patients with functional Univentricular heart after Fontan's surgery and healthy controls: measurement by 4D flow magnetic resonance imaging and streamline visualization. Congenit Heart Dis. 2017;12(1):40-8.

18. Sjoberg P, Heiberg E, Wingren P, Ramgren Johansson J, Malm T, Arheden $\mathrm{H}_{\text {, }}$ et al. Decreased diastolic ventricular kinetic energy in young patients with Fontan circulation demonstrated by four-dimensional cardiac magnetic resonance imaging. Pediatr Cardiol. 2017;38(4):669-80.

19. Ten Harkel AD, Takken T, Van Osch-Gevers M, Helbing WA. Normal values for cardiopulmonary exercise testing in children. Eur I Cardiovasc Prev Rehabil. 2011;18(1):48-54.

20. Kamphuis VP, van der Palen RLF, de Koning PJH, Elbaz MSM, van der Geest RJ, de Roos A, et al. In-scan and scan-rescan assessment of LV in- and outflow volumes by 4D flow MRI versus 2D planimetry. J Magn Reson Imaging. 2018b;47(2):511-22.

21. Elbaz MS, van der Geest RJ, Calkoen EE, de Roos A, Lelieveldt BP, Roest AA, et al. Assessment of viscous energy loss and the association with threedimensional vortex ring formation in left ventricular inflow: in vivo evaluation using four-dimensional flow MRI. Magn Reson Med. 2017;77(2):794-805.

22. Kamphuis VP, Elbaz MSM, van den Boogaard PJ, Kroft LJM, van der Geest RJ, de Roos A, et al. Disproportionate intraventricular viscous energy loss in Fontan patients: analysis by 4D flow MRI. Eur Heart J Cardiovasc Imaging. 2019;20(3):323-33.

23. Kamphuis VP, Westenberg JJM, van der Palen RLF, van den Boogaard PJ, van der Geest RJ, de Roos A, et al. Scan-rescan reproducibility of diastolic left ventricular kinetic energy, viscous energy loss and vorticity assessment using 4D flow MRI: analysis in healthy subjects. Int J Cardiovasc Imaging. 2018c;34(6):905-20.

24. Klein S, Staring M, Murphy K, Viergever MA, Pluim JP. Elastix: a toolbox for intensity-based medical image registration. IEEE Trans Med Imaging. 2010; 29(1):196-205

25. Kheradvar A, Pedrizzetti G. Vortex formation in the heart. In: Vortex formation in the cardiovascular system. London: Springer; 2012. p. 19.

26. Kilner PJ, Henein MY, Gibson DG. Our tortuous heart in dynamic mode--an echocardiographic study of mitral flow and movement in exercising subjects. Heart Vessel. 1997;12(3):103-10.
27. Cesarovic N, Busch J, Lipiski M, Fuetterer M, Fleischmann T, Born S, et al. Left ventricular blood flow patterns at rest and under dobutamine stress in healthy pigs. NMR Biomed. 2019;32(1):e4022.

28. Calkoen EE, Elbaz MS, Westenberg JJ, Kroft LJ, Hazekamp MG, Roest AA, et al. Altered left ventricular vortex ring formation by 4-dimensional flow magnetic resonance imaging after repair of atrioventricular septal defects. J Thorac Cardiovasc Surg. 2015;150(5):1233-40 e1.

29. Gharib MR, Rambod E, Shariff K. A universal time scale for vortex ring formation. J Fluid Mech. 1998;360:121-40.

30. Paterson AR. A first course in fluid dynamics. Cambridge: Cambridge University Press; 1983.

31. Paul IP, Prakash KA, Vengadesan S. Onset of laminar separation and vortex shedding in flow past unconfined elliptic cylinders. Phys Fluids. 2014;26: 023601

32. Negishi K, Borowski AG, Popovic ZB, Greenberg NL, Martin DS, Bungo MW, et al. Effect of gravitational gradients on cardiac filling and performance. J Am Soc Echocardiogr. 2017;30(12):1180-8.

33. Shafer KM, Garcia JA, Babb TG, Fixler DE, Ayers CR, Levine BD. The importance of the muscle and ventilatory blood pumps during exercise in patients without a subpulmonary ventricle (Fontan operation). J Am Coll Cardiol. 2012;60(20):2115-21.

\section{Publisher's Note}

Springer Nature remains neutral with regard to jurisdictional claims in published maps and institutional affiliations.

\section{Ready to submit your research? Choose BMC and benefit from:}

- fast, convenient online submission

- thorough peer review by experienced researchers in your field

- rapid publication on acceptance

- support for research data, including large and complex data types

- gold Open Access which fosters wider collaboration and increased citations

- maximum visibility for your research: over $100 \mathrm{M}$ website views per year

At BMC, research is always in progress.

Learn more biomedcentral.com/submissions 